\title{
Finite Element Analysis of Stress Distribution in Flat and Elevated-Rim Polyethylene Acetabular Liners
}

\author{
Nobuhiro Kaku, MD, Ai Tanaka, MD, Hiroaki Tagomori, MD, Hiroshi Tsumura, MD \\ Department of Orthopaedic Surgery, Faculty of Medicine, Oita University, Yufu, Japan
}

Background: No study has compared flat and elevated-rim polyethylene liners in terms of stress distribution on the bearing surface. The purpose of this study was to investigate the difference in stress distribution between flat and elevated-rim polyethylene liners.

Methods: A stress analysis was performed by using the 3-dimensional finite element method. The cup was placed at an open angle of $20^{\circ}$, the flat liner and the liner with a $10^{\circ}$ elevation was placed at inclination angles of $80^{\circ}, 70^{\circ}$, and $60^{\circ}$.

Results: Compared with the $60^{\circ}$ flat liner, the $80^{\circ}$ and $70^{\circ}$ flat liners showed higher stress at the liner edge. In the elevated-rim liner, the stress was high at the liner edge along the cup edge. When the von Mises equivalent stress was applied to each element of the liner, the high stress area (volume) was the largest for the $80^{\circ}$ flat liner, second largest for the $80^{\circ}$ elevated-rim liner, and third largest for the $70^{\circ}$ flat liner. The average contact pressure also followed the same order.

Conclusions: Elevated-rim liners affect the stress distribution by increasing the area of contact. However, since elevated-rim liners exhibit high stress at the cup edge, they are likely to result in new problems including liner failure. These findings could aid surgeons in the selection of liners and determination of revision methods such as isolated liner exchange vs. acetabular cup revision for a well-fixed metal cup with a higher inclination angle in revision total hip arthroplasty.

Keywords: Elevated-rim liner, Flat liner, Stress distribution, Total hip arthroplasty

Unlike in cemented total hip arthroplasty (THA), several types of polyethylene liners can be used in cementless THA. Selection of the appropriate polyethylene liner affects the long-term stability after THA. ${ }^{1,2)}$ One of the main causes for revision surgery is postoperative dislocation, and the use of elevated polyethylene liners can effectively correct the inclination of metal cups, thereby preventing future dislocations. In cementless THA revision, there are some cases where the metal cup has no looseness, but the polyethylene liner is worn down and progressive osteolysis

Received October 10, 2019; Accepted January 31, 2020

Correspondence to: Nobuhiro Kaku, MD

Department of Orthopaedic Surgery, Faculty of Medicine, Oita University,

1-1 Idaigaoka Hazamacho, Yufu 879-5593, Japan

Tel: +81-97-586-5872, Fax: +81-97-586-6647

E-mail: nobuhiro@oita-u.ac.jp is seen. Significant malalignment of the metal cup requires removal of the metal cup, adjustment of the angle, and reinstallation. However, in cases of mild malalignment of the metal cup, the polyethylene insert may be changed from flat to elevated, with the metal cup preserved, to reduce polyethylene wear and prevent postoperative dislocation; this procedure is also less invasive for patients. ${ }^{3-5)}$ Nevertheless, no studies have compared flat and elevated-rim polyethylene liners in terms of stress distributions on the bearing surface of the liners. Our hypothesis was that the high stress area on the bearing surface would decrease when an elevated-rim polyethylene liner is used rather than a flat liner. By identifying the differences in stress distributions of the 2 types of liners, it may be possible to determine whether the elevated-rim liner is useful for reducing polyethylene wear. Furthermore, this could reveal other valuable practices for the use of elevated-rim liners. 
Kaku et al. Stress Distribution of Acetabular Liners

Clinics in Orthopedic Surgery • Vol. 12, No. 3, $2020 \bullet$ www.ecios.org

In the field of THA, useful information has been obtained from simulation studies using the finite element method $(\mathrm{FEM}) .{ }^{6}$ In this study, we used the FEM to investigate the differences in stress distributions of the flat and elevatedrim polyethylene liners.

\section{METHODS}

We designed a 3-dimensional finite element analysis model. The component models used were the Trinity Acetabular cluster shell with 3 screw holes and the TriFit TS Cementless Tapered Femoral Stem (Corin Ltd., Cirencester, UK), and the linear model used was the ECiMa Vitamin E-stabilized, highly cross-linked ultrahigh molecular weight polyethylene (UHMWPE; Corin Ltd.). The outer diameter of the cup was $50 \mathrm{~mm}$, and the bearing surface diameter of the polyethylene liner was $36 \mathrm{~mm}$. The diameter of the BIOLOX delta ball head (CeramTec, BadenWürttemberg, Germany) was $36 \mathrm{~mm}$ without additional stem neck length. A bone model was created by the extraction and configuration of the bone shape in the pelvis and femur from the computed tomography image of a woman who was 58 years old, which is the mean age of those undergoing THA. The bone model and computed tomography image that we used are owned by the Research Center of Computational Mechanics in the MECHANICAL FINDER Technical Department (Tokyo, Japan).

To make it simpler to evaluate the effect of stress distribution on the elevated-rim liner, the load had to be secured on the elevated rim. Thus, the cup placement angles were fixed at $80^{\circ}, 70^{\circ}$, and $60^{\circ}$, which are larger than the usual operative cup inclination angles; however, the elevated-rim certainly contacted the ball head at each inclination angle in this study. For the flat polyethylene liner, we used $20^{\circ}$ of operative anteversion angle. For the elevat- ed polyethylene liner, the cup placement angles were also fixed at $80^{\circ}, 70^{\circ}$, and $60^{\circ}$ for the operative cup inclination angle and at $20^{\circ}$ for operative anteversion. The elevated polyethylene liner was elevated by $10^{\circ}$ from the cup. The shape of the elevated liner is shown in Fig. 1. The equatorial plane of the flat liner was offset by $1.49 \mathrm{~mm}$ from the plane of the cup. The bearing surface of the flat liner was formed up from the center to the edge with a curvature radius of $18.225^{\circ}$. The elevated surface of the elevated liner was formed in the same direction as the curvature of the bearing, but in flat shape. Thus, to be precise, the curvatures were slightly different between the bearing and the elevated-rim's surface. When the elevated liner was viewed from the bearing surface, the elevation from the equatorial plane has a maximum height of $4 \mathrm{~mm}$ at the center, and when it is viewed from the side, it has an inclination of $10^{\circ}$. In the liner of this model, the center of the elevation rim faced the outermost position with cup inclination and anteversion angle of $0^{\circ}$. From there, it was rotated with an anteversion of $20^{\circ}$ and inclination of $60^{\circ}, 70^{\circ}$, or $80^{\circ}$. The zenith of the elevation rim was facing 12 o'clock.

The models were divided into tetrahedral elements (element size, $0.3-2.5 \mathrm{~mm}$ ) and analyzed. The number of elements in the models was 1,450,000 and the number of nodal points was 280,000 . The Young's moduli and Poisson's ratios were as follows: cup and stem produced from titanium alloy, $109 \mathrm{GPa}$ and 0.28 , respectively; polyethylene liner, $1.00 \mathrm{GPa}$ and 0.46 , respectively; and metal head of cobalt and chromium alloy, $223 \mathrm{GPa}$ and 0.31 , respectively. The Young's modulus of bone was 0.4 , and the value was obtained by using the methods of Keyak and Poisson's ratios. Because the $\mathrm{X}$-axis was defined as the medial to lateral direction, the $\mathrm{Y}$-axis was defined as the anterior to posterior direction, and the $\mathrm{Z}$-axis was defined as the distal to proximal direction. Two thousand $\mathrm{N}$ of force was
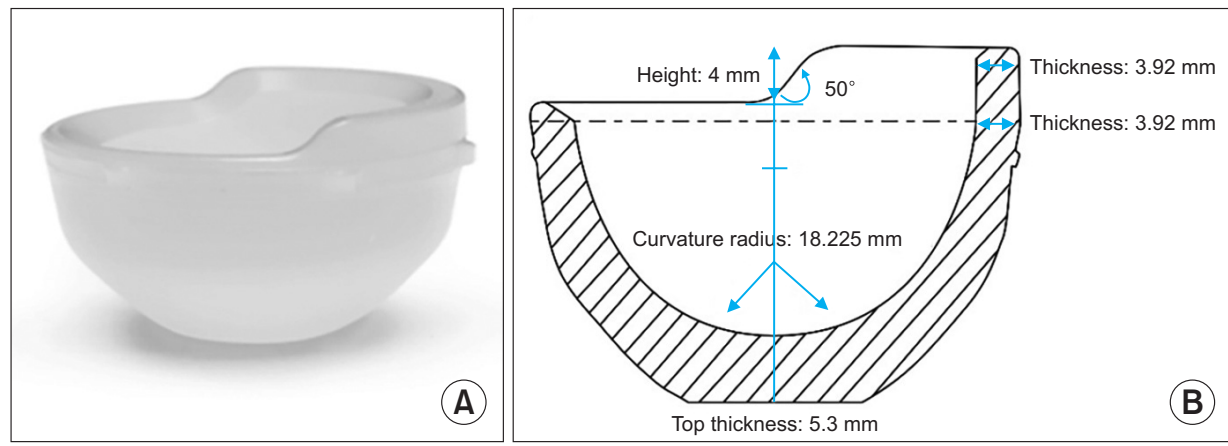

Fig. 1. The shape of an elevated polyethylene liner. (A) A photograph of the actual polyethylene liner. It is cross-linked and has a curvature radius of $18.225 \mathrm{~mm}$ in the bearing surface. The liner thickness is $5.3 \mathrm{~mm}$ at the center of the bearing surface. (B) Cross section of the polyethylene liner. The polyethylene thickness is $5.3 \mathrm{~mm}$ at the center of the bearing surface and $3.92 \mathrm{~mm}$ in the elevated part. The shape of the polyethylene liner is elevated at an angle of $50^{\circ}$ from the equator plane of the liner opening and immediately reaches a height of $4 \mathrm{~mm}$. 
Kaku et al. Stress Distribution of Acetabular Liners

Clinics in Orthopedic Surgery • Vol. 12, No. 3, $2020 \bullet$ www.ecios.org

loaded onto the knee joint surface of the femur toward the proximal of the z-direction. To implement a restraint condition, the sacral joint surface was completely restrained, the pubic joint symmetry was confirmed for the yz-plane, and the femoral distal end was restrained (except in the $\mathrm{z}$ direction).

Interfaces were completely fixed by bond fixation between the femur and the stem, between the acetabular bone and the cup, and between the stem and the ball head. The coefficient of friction was set to 0 under contact conditions between the liner and cup or the ball head.
The 3 conditions for each type of liner $-80^{\circ}, 70^{\circ}$, and $60^{\circ}$ of inclination angles-were simulated by using the finite element analysis with the MECHANICAL FINDER ver. 10.0 Extended Edition (Research Center of Computational Mechanics).

\section{RESULTS}

The von Mises equivalent stress distribution on the bearing surface of the liner is shown in Fig. 2. The area of high stress was the largest in the $80^{\circ}$ flat liner, followed in de-
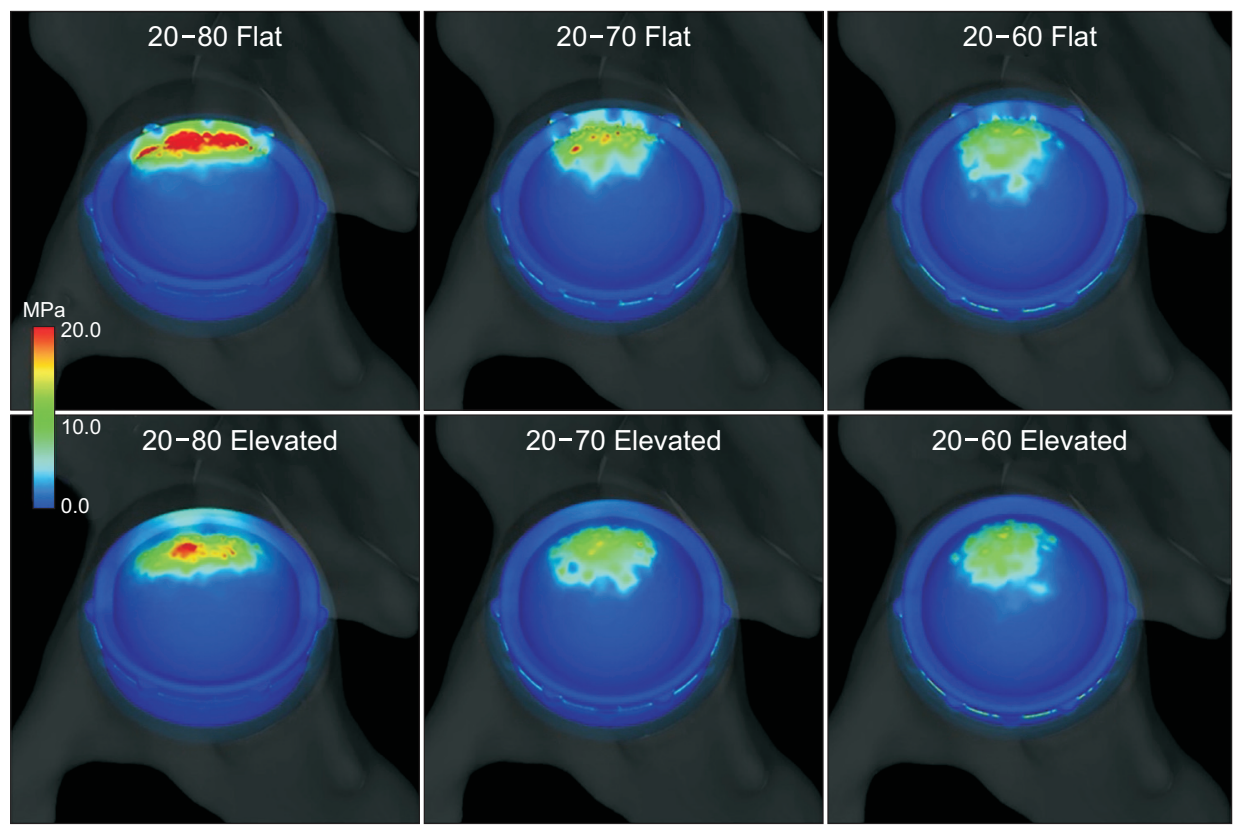

Fig. 2. Equivalent stress on the bearing surface of the polyethylene liner. The von Mises equivalent stress distribution of the polyethylene liner is shown. The flat liner with an inclination angle of $80^{\circ}$ has a wide high stress area concentrated at the edge portion of the liner. In the elevated-rim liner with an inclination angle of $80^{\circ}$, the stress is concentrated inside the edge. The high stress area is narrower in the liners with an inclination angle of $70^{\circ}$.

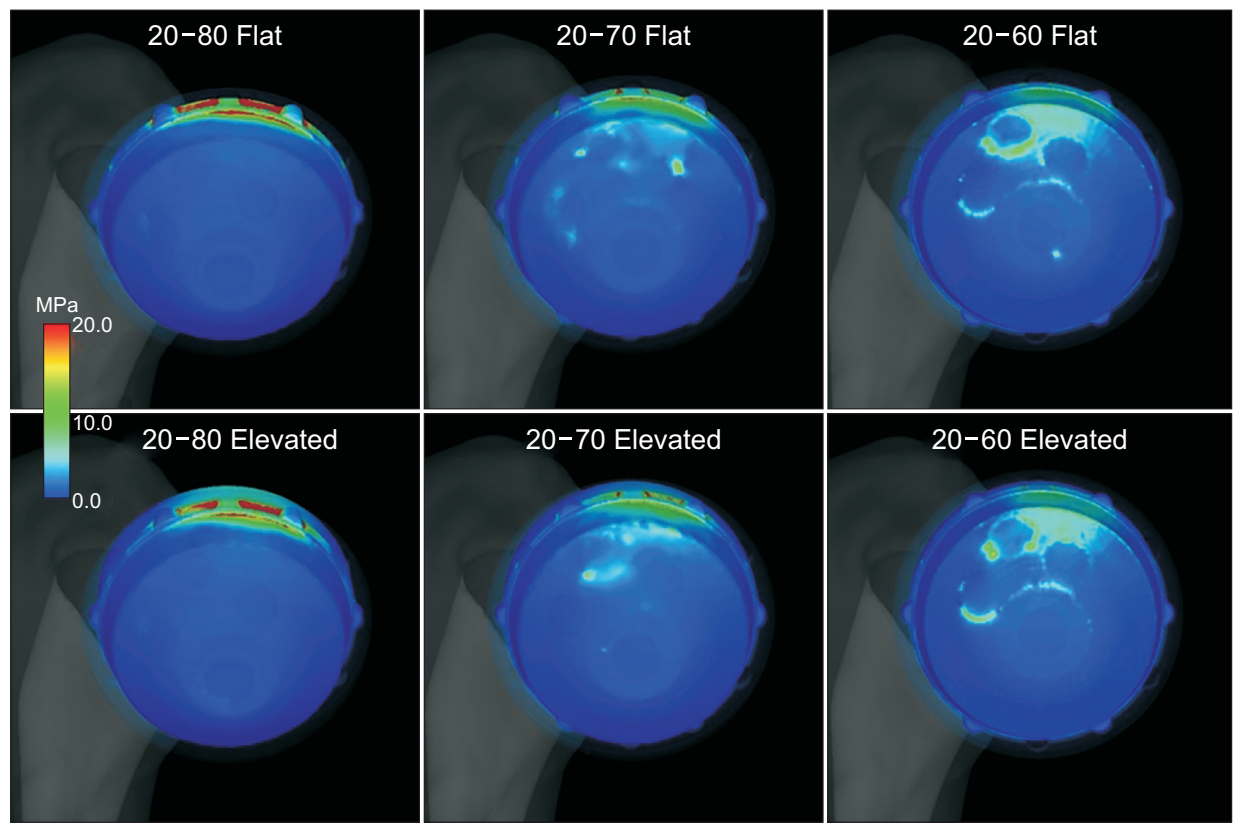

Fig. 3. Equivalent stress on the cup side of the polyethylene liner. The von Mises equivalent stress distribution of the polyethylene liner is shown from the cup side. The results are similar to those of stress distribution on the bearing surface as seen in Fig. 2. 
Kaku et al. Stress Distribution of Acetabular Liners

Clinics in Orthopedic Surgery • Vol. 12, No. 3, $2020 \bullet$ www.ecios.org

scending order by the $80^{\circ}$ elevated-rim liner, $70^{\circ}$ flat liner, and $70^{\circ}$ elevated-rim liner. When the inclination angle of the metal cup was $80^{\circ}$, the flat liner was highly stressed at the edge. Conversely, when the inclination angle of the metal cup was $80^{\circ}$, the $10^{\circ}$ elevated-rim liner was highly stressed away from the edge. The stress was high at the surface corresponding to the metal cup edge, which was more central than the edge of the elevated-rim liner. When the inclination angle of the metal cup was $70^{\circ}$, the areas showing high stress were scarce. From the cup side, the flat liner with an inclination angle of $80^{\circ}$ was highly stressed at the edge, while the elevated-rim liner was highly stressed at the fitting interface with the metal cup (Fig. 3). In all cases, the high stress was spread to the back of the liner. All metal cups had high stress at the edge. The area of high stress was the largest for the $80^{\circ}$ flat liner, followed in descending order by the $80^{\circ}$ elevated-rim liner, and $70^{\circ}$ flat liner (Fig. 4).

The von Mises equivalent stress on each element of the liners is shown in a graph, which depicts the increase in volume every $5 \mathrm{MPa}$ (Fig. 5). The $80^{\circ}$ flat liner had a larger high stress area (volume) than the $80^{\circ}$ elevated-rim liner; the elevated-rim liner had reduced volume compred to that of the flat liner under the same stress. However, the total equivalent stress became extremely small and the difference between flat and elevated-rim liners became very small in the $60^{\circ}$ inclination. The size of the contact area was the smallest for the $80^{\circ}$ flat liner, followed in ascending order by the $80^{\circ}$ elevated-rim liner, and $70^{\circ}$ flat liner. The contact area limited to the elevated-rim liner was 35.5 $\mathrm{mm}^{2}$ in $80^{\circ}$ inclination, $12.8 \mathrm{~mm}^{2}$ in $70^{\circ}$, and $2.6 \mathrm{~mm}^{2}$ in $60^{\circ}$, respectively. The average contact pressure was the highest for the $80^{\circ}$ flat liner, followed in descending order by the $80^{\circ}$ elevated-rim liner, and $70^{\circ}$ flat liner (Table 1 ). Regarding the location of maximum contact pressure, it was found at the edge for flat liners with $70^{\circ}$ and $80^{\circ}$ inclinations; however, for flat liners with $60^{\circ}$ inclination, the maximum contact pressure was found to be closer to the center than the edge (Fig. 6). On the other hand, the maximum contact pressure of the elevated-rim liners was located more centrally than that of the flat liners at any inclinations of the cup.

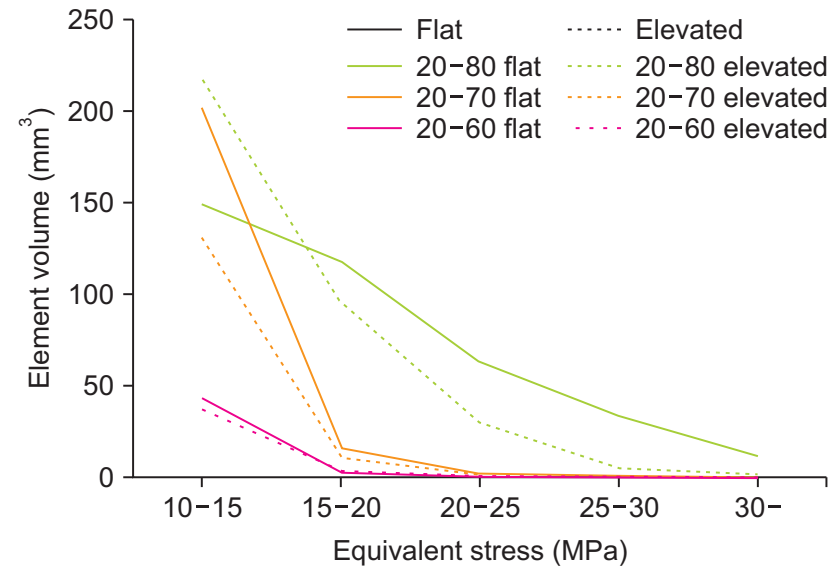

Fig. 5. Element volume for each threshold value of equivalent stress. The graph shows the von Mises equivalent stress at an interval of $5 \mathrm{MPa}$ on each element of a liner. The area (volume) was larger where the stress was higher. The size of the stress area was the largest for the $80^{\circ}$ flat liner, second largest for the $80^{\circ}$ elevated-rim liner, and third largest for the $70^{\circ}$ flat liner.
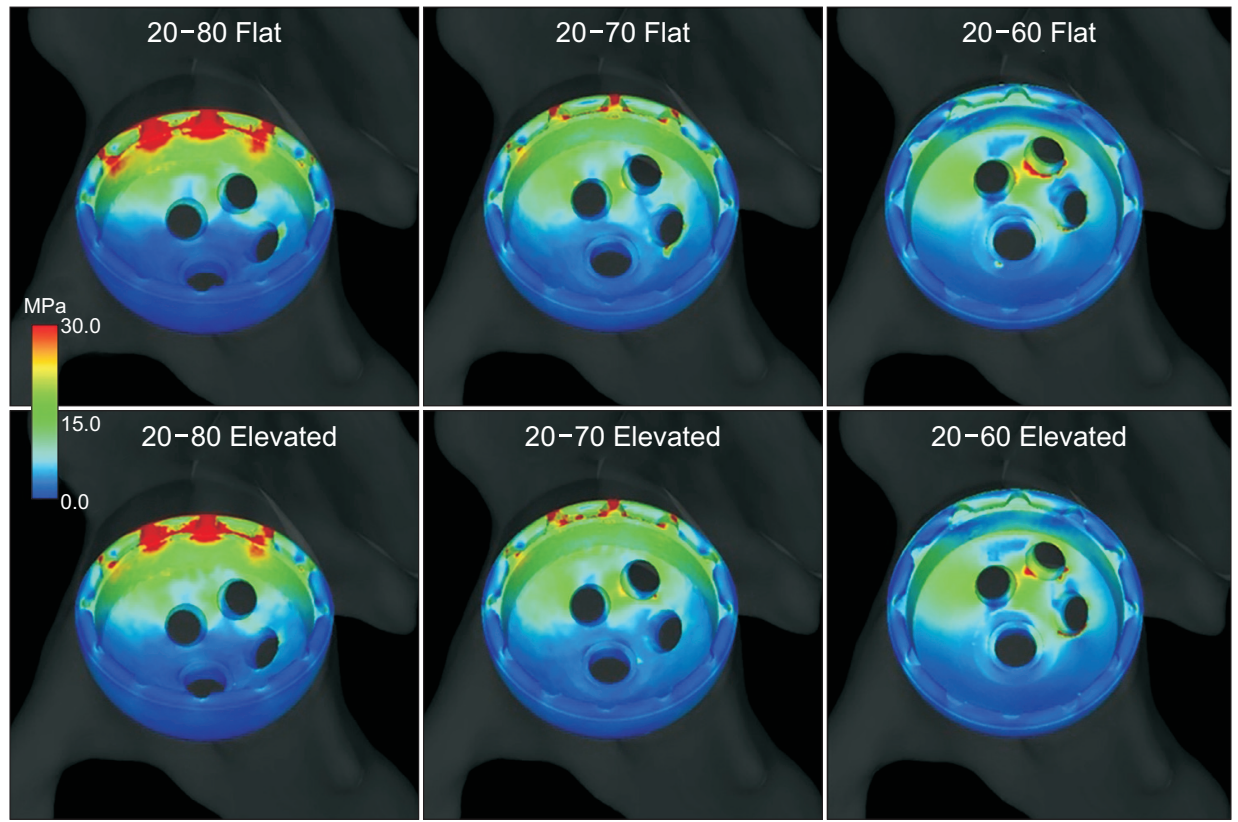

Fig. 4. Equivalent stress on the metal cup. All metal cups have high stress at the edge. The size of the area with high stress is the largest for the $80^{\circ}$ flat liner, second largest for the $80^{\circ}$ elevated-rim liner, and third largest for the $70^{\circ}$ flat liner. 
Table 1. Contact Areas and Contact Pressures

\begin{tabular}{|c|c|c|c|c|c|c|}
\hline \multirow{2}{*}{ Variable } & \multicolumn{2}{|c|}{$20-80$} & \multicolumn{2}{|c|}{$20-70$} & \multicolumn{2}{|c|}{$20-60$} \\
\hline & Flat & Elevated & Flat & Elevated & Flat & Elevated \\
\hline Contact area $\left(\mathrm{mm}^{2}\right)$ & 85.6 & 146.2 & 170.7 & 200.0 & 202.2 & 248.9 \\
\hline Maximum contact pressure (MPa) & 80.9 & 31.9 & 38.8 & 24.2 & 34.1 & 18.4 \\
\hline Average contact pressure (MPa) & 17.9 & 9.7 & 7.8 & 7.1 & 6.7 & 5.8 \\
\hline
\end{tabular}
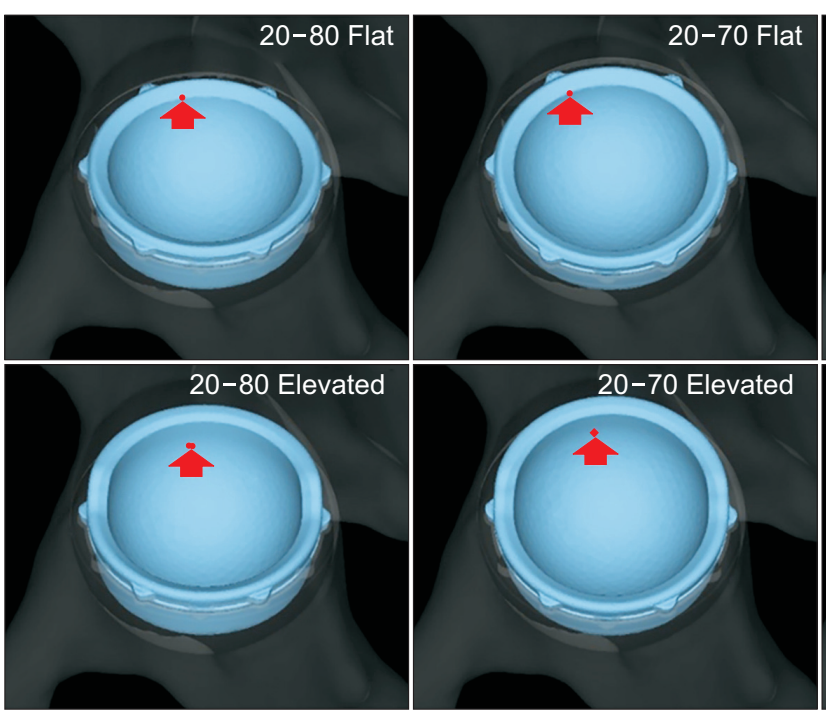

\section{DISCUSSION}

According to our FEM stress analysis, the elevated-rim liners reduced the maximum stress and high stress areas of the bearing surface. The elevated-rim liner increased the load area and dispersed the stress, although the effect of the elevation was proportional to that of the inclination angle of the metal cup. However, the elevated-rim liner has a boundary between the portion where polyethylene is supported by the metal cup and the portion where it is not. This causes deflection of the polyethylene and occurrence of new stress. Thus, the flat liner exhibits high stress at the edge of the liner, while the elevated-rim liner exhibits high stress in the inner area, which is at the same point as the edge of the metal cup. In view of the load direction, compression is on the back side of the liner and tensile strength, on the bearing surface. Thus, there is a concern that the elevated-rim liner may be damaged. By contrast, the stress when the angle of inclination of the cup is reduced is less than that of the elevated-rim liner when the inclination angle is the same. With respect to polyethylene supported by a metal cup, the boundary was not formed and the effect of reducing the inclination angle was suf-

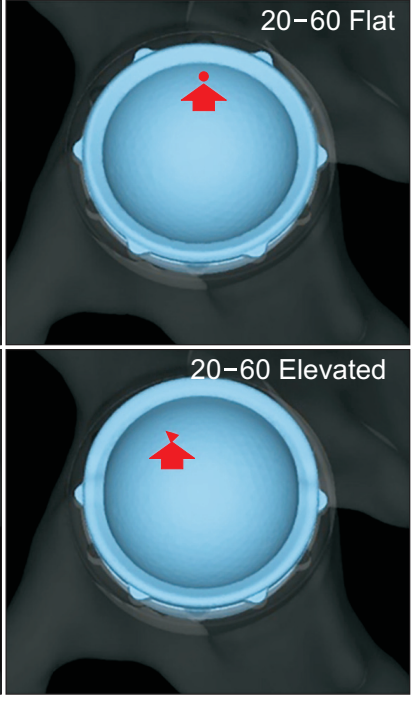

Fig. 6. Location of the maximum contact pressure. The maximum contact pressure of flat liners with $70^{\circ}$ and $80^{\circ}$ inclinations was at the edge, whereas that of the flat liner with $60^{\circ}$ inclination and all elevatedrim liners was more distant from the edge. ficiently exhibited. The rise in stress at this boundary often depends on the shape of the metal cup end and the shape and quality of the polyethylene liner; therefore, these factors should be taken into consideration in the future development and manufacture of implants. Although there are other causes of THA failure such as impingement, cases of failure with elevated-rim polyethylene liners have been reported..$^{7-9)}$

While the contact area was increased and average contact pressure was decreased according to decrease of the cup inclination, the maximum contact pressure of the flat liner with $70^{\circ}$ inclination was higher than that of the elevated-rim liner with $80^{\circ}$ inclination. The same applies to the relationship between the $70^{\circ}$ and $60^{\circ}$ inclinations. When using a flat liner, the metal cup and polyethylene liner have the same edge, so the polyethylene liner is completely lined with metal, and therefore, it cannot be allowed to bend and receive the stress decently. This may be the reason why the maximum contact pressure of the flat liner with $70^{\circ}$ inclination was higher than that of the elevated-rim liner with $80^{\circ}$ inclination, although the influence of the size of the mesh in the FEM analysis could also be a contributing factor. 
The ability of surgeons to select a liner with several variations during the initial surgical treatment with cementless THA is useful for fine adjustment to achieve optimal leg and joint stability. ${ }^{2)}$ Elevated-rim liners can adjust the angle of the liner opening and increase the jumping distance and are used for improving joint stability by preventing dislocation. ${ }^{1)}$ In case of revision with good fixation of the artificial joint, liner replacement is advantageous to the patient and the surgeon: the operative procedure of liner replacement is simple, the surgical invasion is small, and postoperative recovery is quick. ${ }^{10)}$ Some reports have shown that mid- and long-term results of revision with only liner replacement are good; revisions with liner replacements are also effective for wear management. ${ }^{11-13)}$

By contrast, there are reports showing that revision surgery with only liner replacement should be selectively performed and that the mid- and long-term results are affected when liner replacement is performed without such discretion. ${ }^{5,14,15)}$ However, when there is no loosening of the artificial joint and the inclination of the cup is not significantly deviated, but the polyethylene is weakened because of wear, if a flat liner was used in the last operation, then 1 option is to replace it with an elevated-rim liner. By replacing the flat polyethylene liner with the elevated-rim liner, the inclination angle of the liner opening is reduced, and the contact area of the load is increased. However, there is no report on the stress analysis comparing elevated-rim and flat liners. Moreover, there is no report on the mechanism by which the elevated-rim liner changes the stress of the load section or how it differs from that of the inclined metal cup.

Stress analysis studies have reported that the load applied to the bearing surface varies with the inclination of the cup." The larger the inclination angle of the cup, the higher the maximum stress on the liner bearing surface and the wider the region of high stress. Long-term clinical results decline if the inclination angle of the cup is large. ${ }^{16}$ ) However, when the inclination angle of the opening is changed in the elevated-rim liner, the polyethylene elevated-rim liner receives the load of the ball head. If the cup inclination is below the normal range and the contact area between the ball head and polyethylene liner is sufficient, then we regard the presence or absence of an elevated rim has little effect on the stress distribution on the bearing surface. In order to secure the load on the elevated rim, the inclination angles of the cup were set to $80^{\circ}, 70^{\circ}$, and $60^{\circ}$ in this study. Although these are extreme inclination angles, the cup opening angle becomes large with respect to the horizontal plane of the ground and therefore, generally resembles the conditions of this study in the following sit- uations: when a leg length discrepancy or abnormal alignment of the spine is present; and when the coronal plane of the pelvis is inclined proximally on the operated side even if the cup was intraoperatively set to the acetabulum with a normal opening angle area. In addition, we speculate that, during a person's daily life, in vivo stresses often place loads on the polyethylene liner, which are almost equal to the loads used in our simulation. For example, this could occur during hip adduction. Based on the results presented in Fig. 5, it can be understood that the elevated-rim liner is effective for load distribution when the inclination angle of the metal cup is more than $60^{\circ}$. However, the evidence was not sufficient to determine that the criteria for the use of an elevated-rim liner for load distribution on the bearing surface in cementless THA is more than $60^{\circ}$ of cup inclination. Further research is required since we only simulated the standing state in this study and the results may not be applicable to all positions involved in human activities.

The principal limitation of this study is that we only used the same type of elevated-rim liner (increased by $10^{\circ}$ from the equatorial plane). The stress behaviors of elevated-rim liners could be evaluated in more detail by comparing our results with results obtained at different elevation angles, for example, $20^{\circ}$ or $30^{\circ}$. The angle setting of the cup was $80^{\circ}, 70^{\circ}$, and $60^{\circ}$, and revision with metal cup replacement was performed under these conditions in actual clinical practice. However, it could be expected that the elevated-rim was hardly stressed at tilt angles less than $60^{\circ}$ because the contact area limited to the elevatedrim was $2.6 \mathrm{~mm}^{2}$ in $60^{\circ}$ inclination in the present study. In addition, daily movements were not reproduced since the analysis did not consider the ligament and muscle strength. Although we believe that simulation studies for stress analysis under various conditions using a more realistic model are necessary to understand the biomechanical features in greater detail, we also think that the knowledge gained in this study is essential and valuable.

In conclusion, as compared with flat liners, elevatedrim liners can increase the contact area of the load and reduce the areas of maximum and high stress. When using a flat liner, high stress generated by the boundary of support from the metal cup is seen at the edge of the liner. By contrast, when using an elevated-rim liner, high stress is generated at the inner boundary of the elevated-rim liner. The inner boundary is distant from the edge of the elevatedrim liner, resulting in damage to the elevated component. Adjusting the inclination angle of the metal cup is more effective for stress dispersion in elevated-rim and flat liners. Surgeons have to consider a variety of factors, such as 
Kaku et al. Stress Distribution of Acetabular Liners

Clinics in Orthopedic Surgery • Vol. 12, No. 3, $2020 \bullet$ www.ecios.org

age and general condition, when selecting the best surgical procedure, especially in cases of revision THA. Our findings should help surgeons who are considering liner options (e.g., exchange to an isolated liner from a flat liner and elevated orientation) or acetabular cup revision for a well-fixed metal cup with a higher inclination angle.

\section{CONFLICT OF INTEREST}

No potential conflict of interest relevant to this article was reported.

\section{ACKNOWLEDGEMENTS}

We thank the staff of the Research Center of Computational Mechanics in the MECHANICAL FINDER Technical Department for providing support for our simulation study.

\section{REFERENCES}

1. Hau R, Hammeschlag J, Law C, Wang KK. Optimal position of lipped acetabular liners to improve stability in total hip arthroplasty: an intraoperative in vivo study. J Orthop Surg Res. 2018;13(1):289.

2. Krushell RJ, Burke DW, Harris WH. Elevated-rim acetabular components: effect on range of motion and stability in total hip arthroplasty. J Arthroplasty. 1991;6 Suppl:S53-8.

3. Cobb TK, Morrey BF, Ilstrup DM. Effect of the elevated-rim acetabular liner on loosening after total hip arthroplasty. J Bone Joint Surg Am. 1997;79(9):1361-4.

4. Koh KH, Moon YW, Lim SJ, Lee HI, Shim JW, Park YS. Complete acetabular cup revision versus isolated liner exchange for polyethylene wear and osteolysis without loosening in cementless total hip arthroplasty. Arch Orthop Trauma Surg. 2011;131(11):1591-600.

5. Kim KW, Yoo JJ, Kim MN, Kim HJ. Isolated acetabular liner exchange for polyethylene wear and osteolysis with wellfixed metal shell. Clin Orthop Surg. 2019;11(3):270-4.

6. Kaku N, Tabata T, Tsumura H. Influence of cup-center-edge angle on micro-motion at the interface between the cup and host bone in cementless total hip arthroplasty: three-dimensional finite element analysis. Eur J Orthop Surg Traumatol. 2015;25(8):1271-7.

7. Furmanski J, Anderson M, Bal S, et al. Clinical fracture of cross-linked UHMWPE acetabular liners. Biomaterials. 2009;30(29):5572-82.

8. Duffy GP, Wannomae KK, Rowell SL, Muratoglu OK. Fracture of a cross-linked polyethylene liner due to impingement. J Arthroplasty. 2009;24(1):158.

9. Kaku N, Tabata T, Tagomori H, Abe T, Tsumura H. The mechanical effects of cup inclination and anteversion angle on the bearing surface. Eur J Orthop Surg Traumatol. 2018;28(1):65-70.

10. Archibeck MJ, Junick DW, Cummins T, Carothers J, White RE Jr. Polyethylene exchange in a second-generation cementless acetabular component. J Arthroplasty. 2009;24(6 Suppl):69-72.

11. Petis SM, Kubista B, Hartzler RU, Abdel MP, Berry DJ. Polyethylene liner and femoral head exchange in total hip arthroplasty: factors associated with long-term success and failure. J Bone Joint Surg Am. 2019;101(5):421-8.

12. Gu X, He J, Tang Y, Zheng Y. Comparison of polyethylene wear before and after hip revision with liner exchange fixed with the original locking mechanism. PLoS One. 2016;11(12):e0167607.

13. Talmo CT, Kwon YM, Freiberg AA, Rubash HE, Malchau H. Management of polyethylene wear associated with a wellfixed modular cementless shell during revision total hip arthroplasty. J Arthroplasty. 2011;26(4):576-81.

14. Restrepo C, Ghanem E, Houssock C, Austin M, Parvizi J, Hozack WJ. Isolated polyethylene exchange versus acetabular revision for polyethylene wear. Clin Orthop Relat Res. 2009;467(1):194-8.

15. Vadei L, Kieser DC, Frampton C, Hooper G. Survivorship of total hip joint replacements following isolated liner exchange for wear. J Arthroplasty. 2017;32(11):3484-7.

16. Georgiades G, Babis GC, Kourlaba G, Hartofilakidis G. Effect of cementless acetabular component orientation, position, and containment in total hip arthroplasty for congenital hip disease. J Arthroplasty. 2010;25(7):1143-50. 